\title{
Vis-A-Wis: Improving Visual Accessibility through Automatic Web Content Adaptation
}

\author{
Giuseppe Santucci \\ Dipartimento di Informatica e Sistemistica \\ Sapienza Università degli studi di Roma \\ santucci@dis.uniroma1.it
}

\begin{abstract}
The accessibility of Web content is gaining an increasing interest and several research activities deal with standards and methodologies for enforcing Web sites accessibility and usability. In spite of all these efforts most of the actual Web sites are still not accessible at all. The reason of that is twofold: from on side, most of Web site developers are unaware of the actual standards and methodologies for accessibility; on the other side, such standards are still too broad to address in an effective way all the accessibility issues. This paper attacks the Web accessibility problem following two different paths: (1) it focuses on a subset of the accessibility issues, i.e., problems associated with hypo-sight and color-blindness, and (2) it provides means for automatically adapting, i.e., transcoding any existing Web page, increasing its accessibility with respect to the aforementioned disabilities.
\end{abstract}

Keywords: Web accessibility, visual impairment; transcoding; assistive technologies, hypo-sight, color-blindness.

\section{Introduction}

Universal access refers to the requirement of coping with diversity in: (1) the characteristics of the target user population (including people with disabilities); (2) the scope and nature of tasks; and (3) the different contexts of use [12]. This paper focuses on a basic universal access requirement: Web content accessibility.

The accessibility of Web content is gaining an increasing interest and several research activities deal with standards and methodologies for enforcing Web sites accessibility (see, e.g., the Web Content Accessibility Guidelines (WCAG) 2.0 [4, 17]). In spite of all these efforts most of the actual Web sites are still not accessible at all. The reason of that is twofold: from on side, most of Web site developers are unaware of the actual standards and methodologies for accessibility; on the other side, such standards are still too broad to address in an effective way all the accessibility issues.

The Vis-A-Wis (VISual Accessibility for Web Interfaces) project attacks the Web accessibility problem following two different strategies:

1. In order to be effective it focuses on a subset of the accessibility issues, dealing with problems associated with hypo-sight and color-blindness. In fact, it is an author belief that, in order to address affectively accessibility issues, it is mandatory to focus on a specific class of users at a time, addressing only the problems that are 
relevant for that class. As an example, while dealing with color-blind people it is crucial to ensure color separation between plain text and hyperlink text; such an activity is totally useless for people impaired by hypo-sight.

2. It defines a set of strategies and metrics to automatically adapt any Web page, according to a specific disability, improving in a measurable way the page accessibility.

It is worth noting that this approach leads to a novel way of evaluating the accessibility of Web sites:

1. accessibility metrics allow for a thinner evaluation capability with respect to, e.g., the ordinal WCAG evaluation conformance scale, A (lowest), AA, and AAA (highest) [17];

2. accessibility metrics allow for identifying specific accessibility flaws, like poor text contrast, wrong text dimension, wrong text color, wrong text font, etc.;

3. accessibility is evaluated separately for any class of users; as an example, a Web page could be accessible for people impaired by hypo-sight and not accessible for color-blind people or vice versa.

Summarizing, the contribution of the paper is the following:

1. It characterizes accessibility in a thinner way, focusing on a subset of accessibility issues a a time (i.e., hypo-sight and color-blindness);

2. It proposes an automatic adaptation strategy for the aforementioned disabilities to make accessible, in principle, any existing Web site;

3. The metrics it introduces allows for evaluating the accessibility of a Web page in a more complete and useful way.

The paper is organized as follows. Section 2 presents related work, Section 3 introduces the metrics we use for evaluating accessibility, associating them to the disabilities we consider in this paper, Section 4 describes the adaptation strategies we use to improve the accessibility and the prototype we developed to test our approach, and Section 5 presents some conclusions and future work.

\section{Related Work}

To the best of our knowledge, the idea of automatically adapt a Web page with respect to a specific disability using accessibility metrics is quite novel. Most of the available proposals present usability definitions and strategies for designing accessible pages, relying mainly on general principles and using few objective parameters (metrics). Some tools, e.g., the Mozilla accessibar plug-in [20] allows for manually alter a Web page, leaving to the user the burden of choosing, the right text color and, for each page, the right text size and line spacing, adopting very simple strategies, like proportional zoom. The same happens for other widely used tools, like, e.g., ZoomText [21]. Other proposals allow for automatic adaptation, called transcoding, but either are intended for mobile devices (see, e.g., [19]) or address different disabilities, e.g. blind people [3]; moreover, most of the transcoding proposals are based on some annotations, e.g., [5], while our system does make any assumption on the pages that it is transcoding. 
In the following we present some accessibility definitions together with strategies and guidelines to produce accessible Web pages.

A first definition of accessibility is in the ISO/IEC Guide 71 [6] that defines Accessible Design as "design focused on principles of extending standard design to people with some type of performance limitation to maximize the number of potential customers who can readily use a product, building or service". Conversely, ISO 9241171 [7] and 9241-20 [8] define accessibility in a very different way as "usability of a product, service, environment or facility by people with the widest range of capabilities", introducing a tight connection with usability.

Shneiderman instead $[10,11]$ proposes "universal usability" as a term to encompass both accessibility and usability, but notes that "access is not sufficient to ensure successful usage", in such a way Shneiderman defines a different ranking of accessibility in comparison to usability: accessibility is a first but not sufficient requirement to achieve universal usability.

More close to the work presented in this paper, the Web Accessibility Initiative (WAI), founded by the World Wide Web Consortium (W3C), gives a widely accepted general definition of Web accessibility as "accessibility means that people with disabilities can perceive, understand, navigate, and interact with the Web, and that they can contribute to the Web. Web accessibility is intended also for older people with changing abilities due to aging". Moreover, the W3C Web Content Accessibility Guidelines (WCAG) 2.0 [4] contains useful accessibility guidelines and metrics that inspired the work presented in this paper.

However, Theofanos [14], states that meeting the required accessibility standards does not, necessarily mean that a Web site is accessible or usable for people with disabilities. On the other hand, Wegge and Zimmermann [18] demonstrates a relationship between the conformance to WCAG and the level of usability of a website while Tathcer et al. [13] state that if a site is accessible then it is even more usable, claiming that accessibility is a subset of usability, meaning that accessibility problems are particular types of usability problems.

To this respect we strongly agree we the Petrie claim [9] that "some problems appear to only affect people with specific disabilities. For example, having a "submit" button with green text on a red background will not pose any problems for people with full color vision, but will be a catastrophic problem for people with red-green color vision deficiency. So not all accessibility problems affect non-disabled users, and are therefore not within the scope of usability problems."

Summarizing, the work presented in this paper differs from the above proposals for three main aspects:

1. accessibility is considered not as a whole but taking into account different aspects relevant to specific user classes;

2. accessibility is evaluated through objective metrics that capture specific accessibility issues;

3. the main perspective is transcoding, i.e., to automatically adapt existing pages addressing specific accessibility issues; moreover the transcoding process does not require additional information (i.e., annotation). 


\section{Usability Metrics}

This section presents a first set of metrics we currently use to evaluate a Web page accessibility; to this aim we first provide some details about the disabilities we address in the paper.

We consider two main classes of users: people impaired by hypo-sight and colorblind people. More precisely we consider three hypo-sight levels:

1. slight hypo-sight: visual residual between $2 / 10$ and $3 / 10$;

2. medium hypo-sight : visual residual between $1 / 10$ and $2 / 10$;

3. serious hypo-sight : visual residual between $1 / 20$ and $1 / 10$;

and four kinds of color-blindness:

1. achromatopsia: only black an white perception;

2. protanopia : red blindness;

3. deuteranopia : green blindness;

4. tritanopia : blue blindness.

From literature analysis and a questionnaire we distributed to more than 150 people we collected some hot issues that might affect a Web page accessibility. We discuss them in the following, presenting, for each of them, a metric able to measure the impact of the actual issue.

\subsection{Text Font}

Web sites very often use poor readable text fonts (e.g., Comics Suns) and several studies exist dealing with the problem of finding the "most readable" font (see, e.g., $[22,15])$. Even if a total order among the various fonts does not exist, it clearly emerges that some fonts, like. e.g., Arial or Verdana perform better than others when readability is a key issue. In order to measure the impact of the font type on accessibility we defined a set RF (Readable Font) that includes, as a starting point Arial and Verdana, and we compute the RFP (Readable Font Proportion) with the following formula:

$\mathrm{RFP}=$ Number of characters in $\mathrm{RF} /$ Total number of characters.

that ranges in $[0 . .1]$ ( 1 is the best value).

\subsection{Text Size}

Besides font choice, the text size is a critical issue for hypo-sight people and we define the $\mathrm{CD}$ value (Critical Dimension) that represents the minimum dimension a text requires in order to be read by people impaired by hypo-sight. It is worth noting that this value depends on the kind of considered hypo-sight (our prototype uses four different values of $\mathrm{CD}$ for the three hypo-sight classes plus the achromatopsia). We compute the RSP (Readable Size Proportion) with the following formula:

$\mathrm{RSP}=$ Number of characters $\geq \mathrm{CD} /$ Total number of characters.

that ranges in [0..1] (1 is the best value). 


\subsection{Text Contrast}

Font and size are not enough and to increase readability we have insure high contrast between text and background. According to Ware [16] luminance ratio is the key parameter to use when measuring contrast and we use the $\mathrm{W} 3 \mathrm{C}$ formula for computing the CR (Contrast Ratio) based on the luminance of text and background $\mathrm{L}_{1}$, and $\mathrm{L}_{2}$, where $\mathrm{L}_{1}>\mathrm{L}_{2}$.

$$
\mathrm{CR}=\left(\mathrm{L}_{1}+0.05\right) /\left(\mathrm{L}_{2}+0.05\right)
$$

where $\mathrm{L}$ is computed weighting the RGB color components with the CIE (Commission Internationale de L'Eclairage) $\mathrm{V}(\lambda)$ function representing the relative sensitivity of the human eye to light of different wavelengths (for the sake of clarity we omit some details about the calculation of R, G, and B values; a full discussion about the matter is in $[16,17])$.

$$
\mathrm{L}=0.2126 * \mathrm{R}+0.7152 * \mathrm{G}+0.0722 * \mathrm{~B}
$$

Using equation (3) we can compute the RCP (Readable Contrast Proportion) as follows:

$\mathrm{RCP}=$ Number of characters having $\mathrm{CR} \geq 5 /$ Total number of characters.

that ranges in [0..1] (1 is the best value) and the threshold 5 is the minimum contrast ratio required by WCAG 2.0 [17].

Obviously, the L calculation differs for color-blind people and in such a case we use a different formula based on the result appearing in [1]. Some details are in the section about color replacement.

As an example of these metrics usage we provide in Figure 1 the evaluation of the author's teaching home page.

If we require a minimum quality level of 0.8 , it is possible to understand that the text size (RSP) is not suitable for medium and serious hypo-sight and that contrast (RCP) is not very high (clear red on white is not a very good choice) for all people but rare trinatopes, more sensible to red. Concerning font usage (RFP) the accessibility is very high: after the first prototype test the author changed his Web site fonts to Arial in a consistent way.

\section{Adaptation Strategies and the Prototype}

In order to describe the automatic adaptation we implement in the prototype (a Mozilla plug-in) we have to introduce two definitions.

- JNTI (just noticeable text increment). JNTI represent the minimum difference in size between two chars that is clearly perceived by a user. Obviously JNTJ is strictly connected with CD (critical dimension) defined in Section 3 and differs for the three hypo-sight classes and achromatopsia.

- JNLS (just noticeable line spacing). JNLS represent the minimum line spacing that allows a user to clearly distinguish two text lines. Obviously JNLS is strictly connected with CD and JNTI and differs for the three hypo-sight classes. 


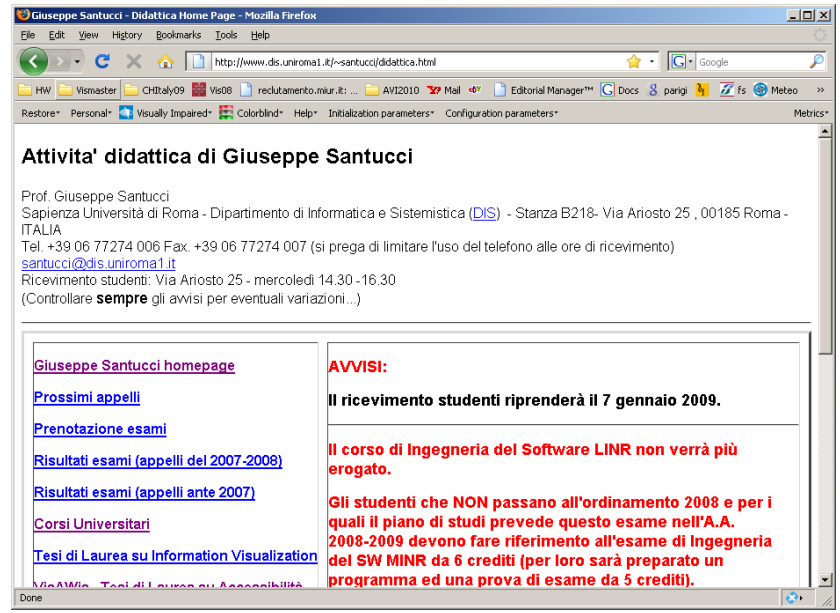

a)

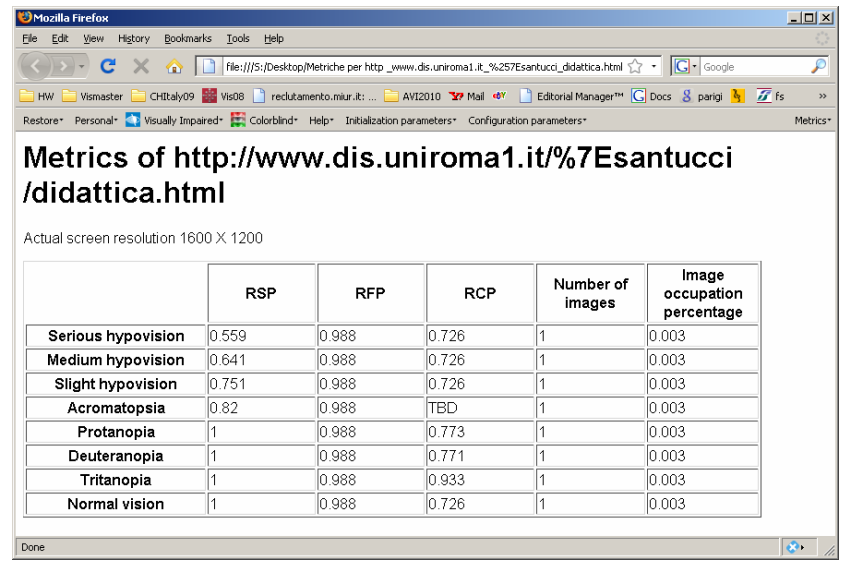

b)

Fig. 1. Accessibility metrics for the author's teaching home page

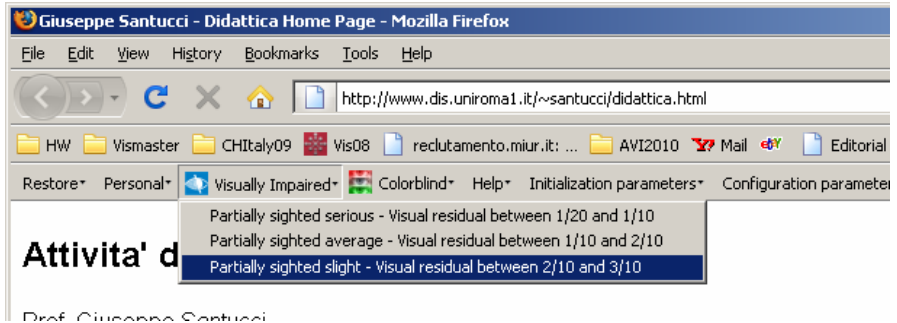

Fig. 2. Selecting the hypo-sight level in the prototype

We implemented two kinds of automatic adaptations: non proportional text size and line space magnification and text/background color replacement. The adaptations 
are automatically applied in a mixed way according the user's disability. The only required action is to declare the disability class s/he belongs to (see the example in Figure 2).

\subsection{Non Proportional Text Magnification}

We use this adaptation for all hypo-sight classes and for achromatopsia (often associated with a very slight hypo-sight). Differently from other proposals that increase text size in a proportional way, we compute an order of all the text sizes available in the page, say $t_{1}, . ., t_{n}$, and we increase $t_{1}$ till CD, $t_{2}$ till CD+JNTI, and so on; line spacing is

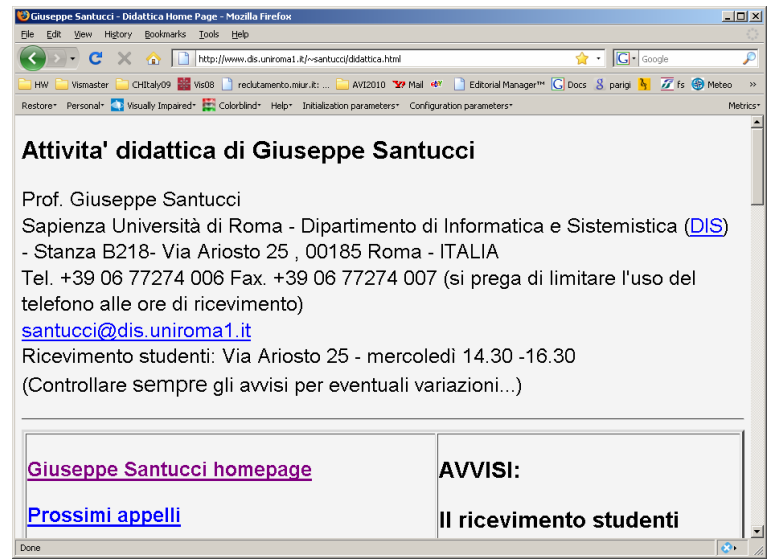

a)

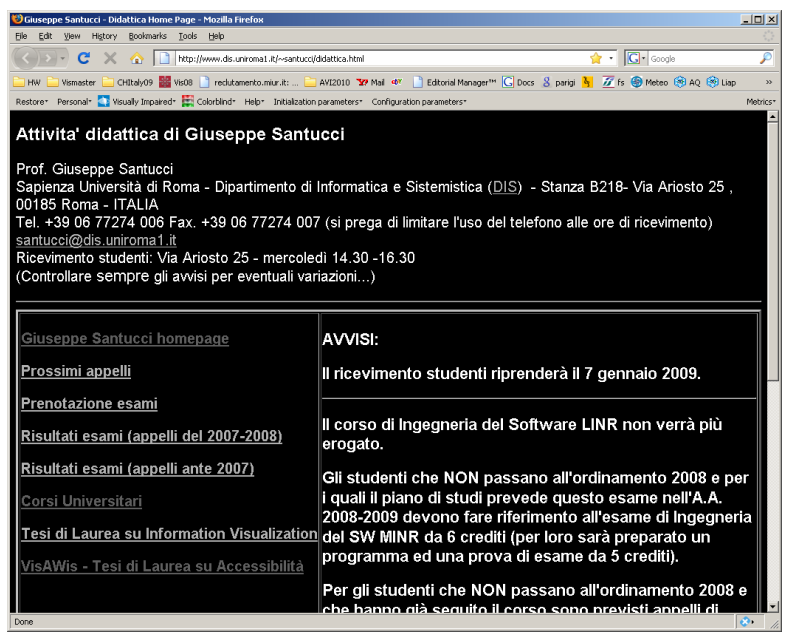

b)

Fig. 3. Adaptation for hypo-sight a) and achromatopsia b) 
increased as well, according to JNLS. In this way we guarantee a high level of readability, maintaining the page structure (as far as text size differences are concerned) maximizing the amount of information that is presented on the screen. Moreover, in order to increase the text contrast we set the foreground to a very light gray (often hypo-sight is associated with a high luminance sensitivity), the font type to Arial and the font color to black.

For achromatopsia (often associated with a very high luminance sensitivity and little hypo-sight) we apply a very little text magnification and we set the background to black and the text color to white. Hyperlinks and visited hyperlinks are rendered with light and dark gray.

Figure 3 shows the adaptation of the page shown in Figure 1 for a) slight hyposight and b) for achromatopsia.

\subsection{Color Replacement}

The main activities a user performs while browsing a Web site are reading text and following hyperlinks, distinguishing visited by non visited ones. To allow dichromatic people to do that we have to alter the color of text, hyperlinks, and visited hyperlinks, in order to guarantee a clear color separation. That corresponds to figure out three different (i.e., perceived as distinct by a color-blind) colors having a high contrast with the background. We shoved in Figure $3 \mathrm{~b}$ the solution for achromatopsia (background: black, text: white, hyperlink: light gray, visited hyperlink: dark gray). To deal with the other kinds of color-blindness we started from the considerations in [16] about the CIE Lu'v' diagrams and we used the simulation described in [12]. The idea is to design a color space in the three-dimensional LMS (Long, Medium, Short wave) space based on the action spectra of the three type of cones pigments as measured at the entrance of the eye and to compute the transformation functions $T$ and $\mathrm{T}^{-1}$ from and to LMS vs. the usual monitor RGB color space. In this way it is possible to transform a color stimulus $\mathrm{Q}$ in the $\mathrm{RGB}$ space in the corresponding $\mathrm{Q}{ }^{\prime}=\mathrm{T} * \mathrm{Q}$ stimulus in the LMS space, remove the value corresponding to the non working cones ( $\mathrm{L}$ for protanopia, M for deuteranopia, and S for trinatopia) obtaining Q', the perceived dichromatic stimulus, and to convert it back to RGB Q" ' $=\mathrm{T}^{-1} * \mathrm{Q}$ "; all the calculations on luminance and color separation are then performed on Q",

Following this approach we devised the following combinations (expressed in term of RGB components):

- Achromatopsia - Already discussed in the Section about text magnification. Background black and text white; hyperlink B0 B0 B0 visited hyperlink 5F 5F 5F;

- Protanopia - Background white and text black; hyperlink 250077 visited hyperlink 5F 73 00;

- Deuteranopia - Background white and text black; hyperlink 1E 0067 visited hyperlink 8553 00;

- Tritanopia - Background white and text black; hyperlink $06356 \mathrm{E}$ visited hyperlink 650207 ; 


\section{Conclusions and Future Work}

This paper presents a novel approach to Web accessibility for person impaired by hypo-sight and color-blindness. The main idea is to adapt (i.e., transcode) in automatic way the content of existing Web pages in order to address these kinds of disability. Transcoding is driven by a suitable set of metrics and mainly consist in altering text font, size, spacing, and color preserving, as much as possible, the original page structure and maximizing the information presented on the screen. A prototype, available at [23], has been implemented to test the adaptation strategies.

The work is still in early stage and, at time of writing, serious user studies have not been performed. The prototype has been demonstrated at Handymatica 2008 [24], the most important Italian event about technologies and handicaps, receiving positive consensus.

We are currently working on:

- Tuning some default values (e.g., JNTI and CD) for the different classes of users we consider;

- Extending and improving the set of accessibility metrics;

- Improving the adaptation algorithm for handling images;

- Setting up a user study for evaluating our approach;

- Deepening the challenging idea of adapting Web pages for blind people, analyzing and adapting the content of a Web page, extending the ideas presented in [2] and [19].

\section{References}

1. Brettel, H., Viénot, F., Mollon, J.D.: Computerized simulation of color appearance for dichromats. J. Opt. Soc. Am. A 14, 2647-2655 (1997)

2. Catarci, T., De Giovanni, L., Gabrielli, S., Kimani, S., Mirabella, V.: Scaffolding the Design of Accessible eLearning Content: a User-Centred Approach \& Cognitive Perspective. Cognitive Processing Journal 9, 209-216 (2008)

3. Fernandes, A., Carvalho, A., Almeida, J., Simões, A.: Transcoding for Web Accessibility for the Blind: Semantics from Structure. In: Proceedings ELPUB 2006 Conference on Electronic Publishing, Bansko, Bulgaria (June 2006)

4. Henry, S.L.: Introduction to Web accessibility (2005), http: / /www.w3 .org/wai/intro/accessibility.php

5. Hori, M., Kondoh, G., Ono, K., Hirose, S.I., Singhal, S.: Annotation-Based Web Content Transcoding. In: Proceedings of the 9th International World Wide Web Conference, Amsterdam, Netherlands (1999)

6. ISO/IEC Guide 71: Guidelines for standards developers to address the needs of older persons and persons with disabilities. International Organization for Standardization, ISO (2001)

7. ISO 9241-171: Ergonomics of human-system interaction - Part 171: Guidance on software accessibility (2006)

8. ISO 9241-20: Ergonomics of human-system interaction - Part 20: Accessibility guidelines for information/ communication technology (ICT) equipment and services (2006)

9. Petrie, H., Kheir, O.: The relationship between accessibility and usability of websites. In: CHI 2007: Proceedings of the SIGCHI conference on Human factors in computing systems, pp. 397-406. ACM Press, USA (2007) 
10. Shneiderman, B.: Universal usability. Communications of the ACM 43(5), 85-91 (2000)

11. Shneiderman, B.: Promoting universal usability with multi-layer interface design. In: Proceedings of the 2003 Conference on Universal Usability, CUU 2003 (2003)

12. Stephanidis, C., Savidis, A.: Universal Access in the Information Society: Methods, Tools and Interaction Technologies. Universal Access in the Information Society Journal, 40-55 (2001)

13. Thatcher, J., Waddell, C.D., Henry, S.L., Swierenga, S., Urban, M.D., Burks, M., Regan, B., Bohman, P.: Constructing accessible Web sites. Glasshaus, San Francisco (2003)

14. Theofanos, M.F., Redish, J.: Bridging the gap: between accessibility and usability. ACM Interactions 10(6), 36-51 (2003)

15. Tullis, T.S., Boynton, J.L., Hersh, H.: Readability of Fonts in the Windows Environment. In: Proc. of the ACM/SIGCHI Conference on Human Factors in Computing, Denver, Colorado, USA, May 7 - 11 (1995)

16. Ware, C.: Information Visualization - Perception for design. Morgan Kaufmann, San Francisco (1999)

17. Web Content Accessibility Guidelines (WCAG) 2.0 (December 11, 2008), http: / / www.w3 . org/TR/WCAG2 0 /

18. Wegge, K.P., Zimmermann, D.: Accessibility, Usability, Safety, Ergonomics: Concepts, Models, and Differences. In: Stephanidis, C. (ed.) HCI 2007. LNCS, vol. 4554, pp. 294 301. Springer, Heidelberg (2007)

19. Yonghyun, H., Jihong, K., Eunkyong, S.: Structure-aware Web transcoding for mobile devices. IEEE Internet Computing 7(5), 14-21 (2003) (transcoding mobile)

20. https://addons.mozilla.org/en-US/firefox/addon/ 4242

21. http://www.synapseadaptive.com/aisquared/zoomtext_9/ zoomtext_9_magnifier_reader.htm

22. http://www.surl.org/

23. http://www.dis.uniroma1.it/ santucci/visawis.html

24. http://www.handimatica.it/ 